Tohoku Math. J.

58 (2006), 259-275

\title{
LOCAL COHOMOLOGY MODULES OF A-HYPERGEOMETRIC SYSTEMS OF COHEN-MACAULAY TYPE
}

\author{
GO OKUYAMA
}

(Received June 21, 2004, revised April 27, 2005)

\begin{abstract}
We study the structure of the local cohomology modules of the Fourier transform of $A$-hypergeometric systems. In particular, we are interested in local cohomology modules with respect to the orbit of a certain action on the toric variety determined by $A$. The purpose in this paper is to describe their structure by using a certain combinatorial object.
\end{abstract}

1. Introduction. Let $K$ be a field of characteristic zero, $A=\left\{\boldsymbol{a}_{1} \cdots \boldsymbol{a}_{n}\right\} \subset \boldsymbol{Z}^{d}$ integral vectors, and $\beta \in K^{d}$. With these data, Gel'fand et al. [7] associated a system of partial differential equations (denoted by $M_{A}(\boldsymbol{\beta})$ ) called an $A$-hypergeometric system. Under the homogeneity condition (see (27)), they determined the characteristic cycles [7] and proved the irreducibility of the monodromy representation for non-resonant parameters [6] in the CohenMacaulay case; Adolphson [1] proved that an $A$-hypergeometric system is holonomic for any $\boldsymbol{\beta}$ without the homogeneity condition, and the holonomic rank of an $A$-hypergeometric system is equal the volume of the convex hull of $\boldsymbol{a}_{1} \cdots \boldsymbol{a}_{n} \in \boldsymbol{Z}^{d}$ in the semi-non-resonant case; Kashiwara proved that an $A$-hypergeometric system is a regular homogeneous holonomic system under the homogeneity condition (cf. Hotta [8]); Saito and Traves [12, 13] classified the parameters according to the $D$-isomorphism classes of their corresponding $A$ hypergeometric systems in combinatorial terms; and many authors studied other $D$-invariants of the A-hypergeometric systems.

We easily see that the Fourier transform of an $A$-hypergeometric system $\mathcal{F}\left(M_{A}(\boldsymbol{\beta})\right)$ has support in the affine toric variety determined by $A$ (denoted by $\mathrm{V}\left(I_{A}(\boldsymbol{x})\right)$ ). The algebraic torus $\left(K^{\times}\right)^{d}$ acts on the affine toric variety $\mathrm{V}\left(I_{A}(\boldsymbol{x})\right)$ through $A$ (see (3)). The orbits of the action can be described in the faces of the convex (rational) polyhedral cone $\boldsymbol{Q}_{\geq 0} A$ generated by $A$, and there exists a one-to-one correspondence between the orbits and the faces (see [5]). Furthermore, it is known that the solution sheaf of $\mathcal{F}\left(M_{A}(\boldsymbol{\beta})\right)$ has an algebraic stratification corresponding to the orbits (see [7,8]). In this paper, we examine the structure of the algebraic local cohomology modules of $\mathcal{F}\left(M_{A}(\boldsymbol{\beta})\right)$ with respect to each orbit (or face) for an arbitrary parameter $\boldsymbol{\beta}$ in the Cohen-Macaulay case. For each face $\sigma$, we see that the local cohomology module with respect to $\sigma$ is isomorphic to a direct sum of the external tensor products of the $D$-modules generated by Dirac's delta function and the algebraic local cohomology modules of $\mathcal{F}\left(M_{A \cap \sigma}(\boldsymbol{\beta}-\boldsymbol{\lambda})\right)$ for some shifted parameters $\boldsymbol{\beta}-\boldsymbol{\lambda}$. We denote by $\boldsymbol{Z} A$ the lattice generated

\footnotetext{
2000 Mathematics Subject Classification. Primary 13D45; Secondary 20M25, 14M25, 13C14, 16S36, 33C70, $42 \mathrm{~A} 38$.

Key words and phrases. Local cohomology, toric varieties, semigroup rings, Cohen-Macaulay modules, Fourier transform, A-hypergeometric systems.
} 
by $A$, and define the equivalence class $\tilde{E}_{\sigma}$ in $\boldsymbol{Z} A$ (see Section 3.2). Then we see that the structure of the algebraic local cohomology modules of $\mathcal{F}\left(M_{A}(\boldsymbol{\beta})\right)$ is completely controlled by $\tilde{E}_{\sigma}$ and $\boldsymbol{\beta}$. Moreover, as an application of this result, we give the structure of the solution sheaf of $\mathcal{F}\left(M_{A}(\boldsymbol{\beta})\right)$ under the homogeneity condition. Since Hotta and Kashiwara [9] gave a relation between the solution of a homogeneous coherent $D$-module and that of its Fourier transform (see Theorem 4.5), we give an approach to knowing the structure of the solution sheaf of the original system and its monodromy representations in detail.

In Section 2, we define some notation and review some necessary notions to argue the local cohomology. To make the computation of the cohomology easier, in Section 3.1, we introduce a double complex associated with the Koszul complex defined in [7] and the Čech complex defined in Section 2. In Section 3.2, we deform the modules appearing in the double complex defined in Section 3.2. In Section 3.3, we give the main theorem in this paper (Theorem 3.12). The keys to the proof of the main theorem are Proposition 3.7 and the acyclicity of the Koszul complex under the Cohen-Macaulay condition (see [2, 14]). In Section 4.1, we provide some examples of the Cohen-Macaulay case. In Section 4.2, we compute the solution sheaf of the Fourier transform of an $A$-hypergeometric system under the Cohen-Macaulay and homogeneity condition.

\section{Preliminaries.}

2.1. $\boldsymbol{A}$-hypergeometric system $\boldsymbol{M}_{\boldsymbol{A}}(\boldsymbol{\beta})$. Let $K$ be a field of characteristic zero, and let

$$
D=K\langle\boldsymbol{x}, \boldsymbol{\partial}\rangle=K\left\langle x_{1}, \ldots, x_{n}, \partial_{1}, \ldots, \partial_{n}\right\rangle
$$

be the $n$th Weyl algebra over $K$. Let $A=\left(\boldsymbol{a}_{1} \cdots \boldsymbol{a}_{n}\right)=\left(a_{i j}\right)$ be a $d \times n$ integer matrix of rank $d$. We denote by $I_{A}(\partial)$ the toric ideal in $K[\partial]$, that is

$$
I_{A}(\boldsymbol{\partial}):=\left\langle\boldsymbol{\partial}^{u}-\partial^{v} \mid A \boldsymbol{u}=A \boldsymbol{v}, \boldsymbol{u}, \boldsymbol{v} \in \boldsymbol{N}^{n}\right\rangle \subset K[\boldsymbol{\partial}] .
$$

Let $\boldsymbol{\beta}={ }^{\mathrm{t}}\left(\beta_{1}, \ldots, \beta_{d}\right) \in K^{d}$ be a parameter (column) vector. We denote by $H_{A}(\boldsymbol{\beta})$ the left ideal of $D$ generated by $I_{A}(\partial) \cup\left\{\sum_{j=1}^{n} a_{i j} x_{j} \partial_{j}-\beta_{i} \mid i=1, \ldots, d\right\}$, and call the quotient module $M_{A}(\boldsymbol{\beta}):=D / H_{A}(\boldsymbol{\beta})$ the $A$-hypergeometric system with parameter $\boldsymbol{\beta}$. Adolphson [1] proved that $M_{A}(\boldsymbol{\beta})$ is a holonomic $D$-module for any $\boldsymbol{\beta}$ without the homogeneity condition (27).

In this paper, we consider the Fourier transform of $M_{A}(\boldsymbol{\beta})$ (denoted by $\mathcal{F} M_{A}(\boldsymbol{\beta})$ ); that is,

$$
\begin{aligned}
\mathcal{F} M_{A}(\boldsymbol{\beta}) & =D / \mathcal{F}^{-1} H_{A}(\boldsymbol{\beta}) \\
& =D / D\left\langle I_{A}(\boldsymbol{x}), \sum_{j=1}^{n} a_{i j} \partial_{j} x_{j}+\beta_{i} \mid i=1, \ldots, d\right\rangle,
\end{aligned}
$$

where $\mathcal{F}$ is the automorphism of $D$ defined by $\mathcal{F}\left(x_{j}\right)=\partial_{j}, \mathcal{F}\left(\partial_{j}\right)=-x_{j}$, and $I_{A}(\boldsymbol{x}):=$ $\mathcal{F}^{-1} I_{A}(\partial)$. It is well-known that a module $M$ over the Weyl algebra is holonomic if and only if $\mathcal{F} M$ is holonomic, and homogeneous regular holonomic if and only if $\mathcal{F} M$ is homogeneous regular holonomic. Here we say that a coherent $D$-module is homogeneous, if for any $m \in M$, $K\left[\sum_{j=1}^{n} x_{j} \partial_{j}\right] m$ is a finite-dimensional $K$-vector space (see $\left.[8,9]\right)$. Moreover, it is clear that 
$\mathcal{F} M_{A}(\boldsymbol{\beta})$ has support in the affine toric variety:

$$
\mathrm{V}\left(I_{A}(\boldsymbol{x})\right):=\left\{\boldsymbol{x} \in K^{n} \mid f(\boldsymbol{x})=0 \text { for all } f \in I_{A}(\boldsymbol{x})\right\} .
$$

Namely,

$$
\operatorname{Supp}\left(\mathcal{F} M_{A}(\boldsymbol{\beta})\right) \subset \mathrm{V}\left(I_{A}(\boldsymbol{x})\right) .
$$

2.2. Affine semigroup algebra $K[N A]$. In this section, let us recall affine semigroup algebras. We also denote the set $\left\{\boldsymbol{a}_{1}, \ldots, \boldsymbol{a}_{n}\right\}$ by $A$ and the affine semigroup generated by $A$ by $N A$. Let $K[N A]$ be the affine semigroup algebra of $N A$; that is,

$$
K[N A]=\bigoplus_{\lambda \in N A} K t^{\lambda} \subset K\left[t_{1}^{ \pm}, \ldots, t_{d}^{ \pm}\right]
$$

as $K$-vector spaces, where $t^{\lambda}:=t_{1}^{\lambda_{1}} \cdots t_{d}^{\lambda_{d}}$, and the multiplication of $K[N A]$ is defined by $\boldsymbol{t}^{\lambda} \cdot \boldsymbol{t}^{\lambda^{\prime}}:=\boldsymbol{t}^{\lambda+\lambda^{\prime}}$. We recall that the toric ideal $I_{A}(\boldsymbol{x})$ is simply the kernel of the $K$-algebra epimorphism

$$
\phi_{A}: K[x] \rightarrow K[N A], \quad \phi_{A}\left(x_{j}\right):=\boldsymbol{t}^{\boldsymbol{a}_{j}} .
$$

We regard $K[N A]$ as a $K[x]$-module through the map $\phi_{A}$; that is, $f \cdot g:=\phi_{A}(f) g(f \in$ $K[x], g \in K[N A])$.

Next we recall a localization of $K[N A]$. We denote by $\boldsymbol{Q}_{\geq 0} A$ the cone $\left\{\sum_{j=1}^{n} c_{j} \boldsymbol{a}_{j} \mid\right.$ $c_{j} \in \boldsymbol{Q}_{\geq 0}$. Let $\mathcal{S}_{A}$ be the set of faces of $\boldsymbol{Q}_{\geq 0} A, \boldsymbol{Z} A$ the group generated by $A$ and $K A$ the $K$-vector space generated by $A$. For a face $\sigma \in \mathcal{S}_{A}$ we denote by $\boldsymbol{Z}(A \cap \sigma)$ the $\boldsymbol{Z}$-submodule of $\boldsymbol{Z} A$ generated by $A \cap \sigma$ and $K(A \cap \sigma)$ the $K$-subspace of $K A$ generated by $A \cap \sigma$. We agree that $\boldsymbol{Z}(A \cap \sigma)=K(A \cap \sigma)=0$ when $A \cap \sigma=\varnothing$. For each $\sigma \in \mathcal{S}_{A}$, we consider two multiplicatively closed sets

$$
S_{\sigma}:=\left\{t^{\lambda} \mid \lambda \in N(A \cap \sigma)\right\}, \quad T_{\sigma}:=\phi_{A}^{-1}\left(S_{\sigma}\right) .
$$

We denote by $K[N A]_{\sigma}$ (resp. $K[x]_{\sigma}$ ) the localization of $K[N A]$ (resp. $K[x]$ ) with respect to $S_{\sigma}$ (resp. $T_{\sigma}$ ). Let $\overline{\phi_{A, \sigma}}$ be the natural morphism induced by $\phi_{A}$. Then we have

$$
\begin{aligned}
K[x]_{\sigma} / K[x]_{\sigma} I_{A}(\boldsymbol{x}) \stackrel{\overline{\phi_{A}}}{\simeq} K[N A]_{\sigma} & =K[N A+\boldsymbol{Z}(A \cap \sigma)] \\
& =\bigoplus_{\lambda \in N A+Z(A \cap \sigma)} K \boldsymbol{t}^{\lambda} .
\end{aligned}
$$

2.3. Orbits of an algebraic torus action on an affine toric variety. To discuss the algebraic local cohomology modules of $\mathcal{F} M_{A}(\boldsymbol{\beta})$, we recall the orbits of the action of the algebraic torus $\left(K^{\times}\right)^{d}$, defined by

$$
\left(K^{\times}\right)^{d} \times \mathrm{V}\left(I_{A}(\boldsymbol{x})\right) \ni\left(\boldsymbol{t}, x_{1}, \ldots, x_{n}\right) \mapsto\left(\boldsymbol{t}^{\boldsymbol{a}_{1}} x_{1}, \ldots, \boldsymbol{t}^{\boldsymbol{a}_{n}} x_{n}\right) \in \mathrm{V}\left(I_{A}(\boldsymbol{x})\right) .
$$

The orbits of the action are the very sets that we consider. In this section, we briefly recall a relationship between the faces of $\boldsymbol{Q}_{\geq 0} A$ and the orbits (cf. [5, 8]).

For $\sigma \in \mathcal{S}_{A}$, we denote by $I_{\sigma}$ the subset $\left\{j \mid \boldsymbol{a}_{j} \in \sigma\right\}$ of $\{1, \ldots, n\}$, and put

$$
X_{\sigma}:=\left\{\boldsymbol{x} \in K^{n} \mid x_{j} \neq 0\left(j \in I_{\sigma}\right), x_{j}=0\left(j \notin I_{\sigma}\right)\right\} \cap \mathrm{V}\left(I_{A}(\boldsymbol{x})\right) .
$$


It is well known that $\left\{X_{\sigma} \mid \sigma \in \mathcal{S}_{A}\right\}$ is the set of orbits. Namely, $X_{\sigma}$ is invariant under the action of $\left(K^{\times}\right)^{d}$ for each $\sigma \in \mathcal{S}_{A}$ and

$$
\mathrm{V}\left(I_{A}(\boldsymbol{x})\right)=\coprod_{\sigma \in \mathcal{S}_{A}} X_{\sigma}, \text { disjoint union } .
$$

Moreover, there exist order-preserving one-to-one correspondences among $\mathcal{S}_{A},\left\{I_{\sigma} \mid \sigma \in \mathcal{S}_{A}\right\}$ and $\left\{\overline{X_{\sigma}} \mid \sigma \in \mathcal{S}_{A}\right\}$, where $\overline{X_{\sigma}}$ is the Zariski closure of $X_{\sigma}$ in $\mathrm{V}\left(I_{A}(\boldsymbol{x})\right)$. Namely,

$$
\begin{gathered}
\mathcal{S}_{A} \longleftrightarrow\left\{I_{\sigma} \mid \sigma \in \mathcal{S}_{A}\right\} \longleftrightarrow\left\{\overline{X_{\sigma}} \mid \sigma \in \mathcal{S}_{A}\right\}, \\
\sigma \longleftrightarrow I_{\sigma} \longleftrightarrow \overline{X_{\sigma}} .
\end{gathered}
$$

2.4. Algebraic local cohomology and $\breve{C e c h}$ complex. We consider the algebraic local cohomology modules with respect to each of the orbits described in Section 2.3. We fix a face $\sigma \in \mathcal{S}_{A}$. Put

$$
L_{\sigma}^{p}:=\bigoplus_{\substack{\sigma<\tau \in \mathcal{S}_{A} \\ \operatorname{dim} \tau-\operatorname{dim} \sigma=p}} K[\boldsymbol{x}]_{\tau} \quad\left(p=0, \ldots, d_{\sigma}:=\operatorname{dim} Q_{\geq 0} A-\operatorname{dim} \sigma\right),
$$

and define $f^{p}: L_{\sigma}^{p} \rightarrow L_{\sigma}^{p+1}$ by specifying its component $f_{\tau^{\prime}, \tau}: K[\boldsymbol{x}]_{\tau^{\prime}} \rightarrow K[\boldsymbol{x}]_{\tau}$ to be

$$
\begin{cases}0 & \text { if } \tau^{\prime} \nprec \tau \\ \varepsilon\left(\tau^{\prime}, \tau\right) \text { nat } & \text { if } \tau^{\prime} \prec \tau\end{cases}
$$

where $\varepsilon$ is a suitable incidence function on $\mathcal{S}_{A}$ and nat is the natural inclusion. It is clear that

$$
\boldsymbol{L}_{\sigma}^{\bullet}: 0 \rightarrow L_{\sigma}^{0} \stackrel{f^{0}}{\longrightarrow} L_{\sigma}^{1} \stackrel{f^{1}}{\longrightarrow} \cdots \stackrel{f^{d^{\sigma}-1}}{\longrightarrow} L_{\sigma}^{d_{\sigma}} \rightarrow 0
$$

is a complex. Then we have the following on the algebraic local cohomology functor $\mathrm{R} \Gamma_{\left[X_{\sigma}\right]}$.

THEOREM 2.1. Let $M$ be a $K[\boldsymbol{x}]$-module with support in $\mathrm{V}\left(I_{A}(\boldsymbol{x})\right)$. Then, for all $\sigma \in \mathcal{S}_{A}$ and for all $p$, we have

$$
H_{\left[X_{\sigma}\right]}^{p}(M) \simeq H^{p}\left(\boldsymbol{L}_{\sigma}^{\bullet} \otimes_{K[x]} M\right) .
$$

Furthermore, if $M$ has a D-module structure, then the tensor product of the D-modules $L_{\sigma}^{p}$ and $M$ has the natural D-module structure, and (6) holds as D-modules.

Proof. Put $Y=\left\{x \in K^{n} \mid x_{j} \neq 0\left(j \in I_{\sigma}\right), x_{j}=0\left(j \notin I_{\sigma}\right)\right\}$. Since $M$ has support in $\mathrm{V}\left(I_{A}(\boldsymbol{x})\right)$, we have $\mathrm{R} \Gamma_{\left[\mathrm{V}\left(I_{A}(\boldsymbol{x})\right)\right]}(M) \simeq M$. Hence, it follows from (4) that

$$
\begin{aligned}
\mathrm{R} \Gamma_{\left[X_{\sigma}\right]}(M) & \simeq \mathrm{R} \Gamma_{[Y]}\left(\mathrm{R} \Gamma_{\left[\mathrm{V}\left(I_{A}(x)\right)\right]}(M)\right) \\
& \simeq \mathrm{R} \Gamma_{[Y]}(M) \\
& \simeq \mathrm{R} \Gamma_{\left[\left\{x_{j}=0 \mid j \in I_{\sigma}\right\}\right]}\left(R \Gamma_{\left[\left\{x_{j}=0 \mid j \notin I_{\sigma}\right\}\right]}(M)\right) \\
& \simeq \mathrm{R} \Gamma_{\left[\left\{x_{j}=0 \mid j \in I_{\sigma}\right\}\right]}\left(K[x]_{\sigma} \otimes_{K[x]} M\right) .
\end{aligned}
$$

Similar to [4, Proposition 3.5.6], we immediately have

$$
H_{\left[\left\{x_{j}=0 \mid j \in I_{\sigma}\right\}\right]}^{p}\left(K[\boldsymbol{x}]_{\sigma} \otimes_{K[x]} M\right) \simeq H^{p}\left(\boldsymbol{L}_{\sigma}^{\bullet} \otimes_{K[x]_{\sigma}} K[\boldsymbol{x}]_{\sigma} \otimes_{K[\boldsymbol{x}]} M\right) \simeq H^{p}\left(\boldsymbol{L}_{\sigma}^{\bullet} \otimes_{K[\boldsymbol{x}]} M\right)
$$

for all $p$. The latter part is clear. 
We call the complex $\boldsymbol{L}_{\sigma}^{\bullet} \otimes_{K[x]} M$ the Čech complex of $M$ associated with $\sigma \in \mathcal{S}_{A}$.

COROLLARY 2.2. For all $\sigma \in \mathcal{S}_{A}$ and all $k$, we have

$$
H_{\left[X_{\sigma}\right]}^{k}\left(\mathcal{F} M_{A}(\boldsymbol{\beta})\right) \simeq H^{k}\left(\boldsymbol{L}_{\sigma}^{\bullet} \otimes_{K[x]} \mathcal{F} M_{A}(\boldsymbol{\beta})\right)
$$

as D-modules.

Proof. This clearly follows from (1) and (6).

3. Main theorem. Throughout this section, assume $A$ to be Cohen-Macaulay; that is, $K[N A]$ is a Cohen-Macaulay algebra. In Section 2.4, we have shown that the algebraic local cohomology modules $H_{\left[X_{\sigma}\right]}^{k}\left(\mathcal{F} M_{A}(\boldsymbol{\beta})\right)$ are computed by the cohomology modules of the Čech complex $\boldsymbol{L}_{\sigma}^{\bullet} \otimes_{K[x]} \mathcal{F} M_{A}(\boldsymbol{\beta})$.

However, it is still difficult to compute them. In Sections 3.1 and 3.2, first we construct another complex, easier to compute, then deform the modules appearing in the complex, and finally give decomposition of them.

In Section 3.3, we state the main result of this paper (Theorem 3.12); that is, an explicit computation of the algebraic local cohomology modules $H_{\left[X_{\sigma}\right]}^{k}\left(\mathcal{F} M_{A}(\boldsymbol{\beta})\right)$.

3.1. Koszul complex. It is still difficult to compute the cohomology modules of the Čech complex $\boldsymbol{L}_{\sigma}^{\bullet} \otimes_{K[x]} \mathcal{F} M_{A}(\boldsymbol{\beta})$ :

$$
0 \rightarrow L_{\sigma}^{0} \otimes \mathcal{F} M_{A}(\boldsymbol{\beta}) \stackrel{f^{0} \otimes \mathrm{id}}{\longrightarrow} \cdots \stackrel{f^{d_{\sigma}-1} \otimes \mathrm{id}}{\longrightarrow} L_{\sigma}^{d_{\sigma}} \otimes \mathcal{F} M_{A}(\boldsymbol{\beta}) \rightarrow 0 .
$$

So, we construct another complex that is easier to compute. To this end, we first recall the Koszul complex $\boldsymbol{M}^{\bullet}:=\operatorname{Kos}^{\bullet}\left(D / D I_{A}(\boldsymbol{x}) ; \sum_{j=1}^{n} a_{i j} \partial_{j} x_{j}+\beta_{i}, i=1, \ldots, d\right)$ (see [2, Section 1] and [14, Section 4.3]),

$$
0 \rightarrow M^{-d} \stackrel{\psi^{-d}(\boldsymbol{\beta})}{\longrightarrow} M^{-d+1} \stackrel{\psi^{-d+1}(\boldsymbol{\beta})}{\longrightarrow} \cdots \rightarrow M^{-1} \stackrel{\psi^{-1}(\boldsymbol{\beta})}{\longrightarrow} M^{0} \rightarrow 0,
$$

defined by

$$
M^{-m}:=\bigoplus_{1 \leqq i_{1}<\cdots<i_{m} \leqq d} D / D I_{A}(\boldsymbol{x}) e_{i_{1}} \wedge \cdots \wedge e_{i_{m}}
$$

and

$$
\begin{aligned}
\psi(\boldsymbol{\beta})^{-m}\left(e_{i_{1}} \wedge \cdots \wedge e_{i_{m}}\right) \\
\quad:=\sum_{k=1}^{m}(-1)^{k}\left(\sum_{j=1}^{n} a_{i_{k} j} \partial_{j} x_{j}+\beta_{i_{k}}\right) e_{i_{1}} \wedge \cdots \wedge \hat{e_{i_{k}}} \wedge \cdots \wedge e_{i_{m}} \quad(i=1, \ldots, d),
\end{aligned}
$$

for $m \in N$. Adolphson [2] showed that, if A is normal (namely $K[N A]$ is a normal algebra), then (9) is acyclic. Namely, the complex

$$
0 \rightarrow M^{-d} \stackrel{\psi^{-d}(\boldsymbol{\beta})}{\longrightarrow} M^{-d+1} \stackrel{\psi^{-d+1}(\boldsymbol{\beta})}{\longrightarrow} \cdots \rightarrow M^{-1} \stackrel{\psi^{-1}(\boldsymbol{\beta})}{\longrightarrow} M^{0} \rightarrow \mathcal{F} M_{A}(\boldsymbol{\beta}) \rightarrow 0
$$

is exact. In other words, (10) gives a certain kind of resolution of $\mathcal{F} M_{A}(\boldsymbol{\beta})$. However, we see that the proof in [2] works under the weaker condition that $A$ is Cohen-Macaulay (namely 
$K[N A]$ is a Cohen-Macaulay algebra). Recall that a normal semigroup algebra is CohenMacaulay.

Proposition 3.1. If A is Cohen-Macaulay, then the Koszul complex (9) is acyclic; that is, the complex (10) is exact.

Proof. This is similar to [2, Theorem 2]. Let $\boldsymbol{t}^{\lambda}:=\overline{\boldsymbol{x}^{\boldsymbol{v}}}$, where $\lambda=A \boldsymbol{v}$. Let $\triangle$ be the convex hull in $\boldsymbol{Q}^{d}$ of $A$ and the origin. We define a filtration on the module $D / D I_{A}(\boldsymbol{x})$ by defining the weight $w\left(t^{\lambda}\right)$ (or $w(\lambda)$ ) of a monomial $t^{\lambda}$ by

$$
w(\boldsymbol{\lambda}):=\inf \left\{w \in \boldsymbol{R}_{\geq 0} \mid \lambda \in w \Delta\right\},
$$

where $w \Delta$ denotes the dilation of $\Delta$ by the factor $w$. We see that there exists a positive integer $e$ such that $w(\lambda) \in e^{-1} N$ for any $\lambda \in N A$. We denote by $F_{m / e}\left(D / D I_{A}(\boldsymbol{x})\right)$ the $K[\boldsymbol{\partial}]-$ module generated by $\boldsymbol{t}^{\lambda}$ with $w(\lambda) \leqq m / e$. Put $g_{i}=\sum_{j=1}^{n} a_{i j} \partial_{j} x_{j} \in F_{1}\left(D / D I_{A}(\boldsymbol{x})\right)$ and let $\bar{g}_{i}$ be its image in the associated graded ring $\operatorname{Gr}\left(D / D I_{A}(\boldsymbol{x})\right)$. Note that $\operatorname{Gr}\left(D / D I_{A}(\boldsymbol{x})\right) \simeq$ $K[\partial][N A]$ is a Cohen-Macaulay ring. Let $\mathcal{K}\left(\operatorname{Gr}\left(D / D I_{A}(\boldsymbol{x})\right),\left\{\bar{g}_{i}\right\}_{i=1}^{d}\right)$ be the Koszul complex on $\operatorname{Gr}\left(D / D I_{A}(\boldsymbol{x})\right)$ formed by $\bar{g}_{1}, \ldots, \bar{g}_{d}$. It is sufficient to show that this complex is acyclic (in positive dimension) as in [2]. Indeed, it follows that the zeroth homology of this complex is of finite rank (see the proof of [1, Lemma 5.2]). So $\left\{\bar{g}_{i}\right\}_{i=1}^{d}$ is a system of parameters for $\operatorname{Gr}\left(D / D I_{A}(x)\right)$. Hence, it follows from the Cohen-Macaulayness of $\operatorname{Gr}\left(D / D I_{A}(\boldsymbol{x})\right)$ that the complex $\mathcal{K}\left(\operatorname{Gr}\left(D / D I_{A}(\boldsymbol{x})\right),\left\{\bar{g}_{i}\right\}_{i=1}^{d}\right)$ is acyclic.

Secondly, let us combine (8) with (10) to make a double complex. Namely, we define a double complex $\boldsymbol{W}^{\bullet, \bullet}:=\left\{W^{p, q}, d_{\mathrm{I}}^{p, q}, d_{\mathrm{II}}^{p, q}(\boldsymbol{\beta})\right\}$ of $D$-modules by

$$
\begin{gathered}
W^{p, q}:=L_{\sigma}^{p} \otimes_{K[x]} M^{q}, \\
d_{\mathrm{I}}^{p, q}=f^{p} \otimes \mathrm{id}, \quad d_{\mathrm{II}}^{p, q}(\boldsymbol{\beta}):=\operatorname{id} \otimes \psi^{q}(\boldsymbol{\beta}) .
\end{gathered}
$$

We note that, for each $\tau \in \mathcal{S}_{A}, T_{\tau}$ (see Section 2.2) satisfies the left and the right Ore conditions in $D$. Thus, the left localization $T_{\tau} D$ and the right localization $D_{T_{\tau}}$ are equal. Hence, it is clear that

$$
\begin{aligned}
& K[x]_{\tau} \otimes_{K[x]} D / D I_{A}(x) \stackrel{\Phi_{l}}{\simeq} D_{T_{\tau}} / D_{T_{\tau}} I_{A}(x) \\
& \stackrel{\operatorname{id} \otimes \overline{\phi_{A}}}{\simeq} D \otimes_{K[x]} K[x]_{\tau} / K[x]_{\tau} I_{A}(\boldsymbol{x}) \\
& \stackrel{\simeq}{\simeq} \otimes_{K[x]} K[N A+Z(A \cap \tau)],
\end{aligned}
$$

where

$$
\Phi_{l}\left(g \otimes P \bmod D I_{A}(\boldsymbol{x})\right)=g P \bmod D I_{A}(\boldsymbol{x}),
$$

and

$$
\Phi_{r}\left(P^{\prime} \otimes g^{\prime} \bmod K[x]_{\tau} I_{A}(x)\right)=P^{\prime} g^{\prime} \bmod D_{T_{\tau}} I_{A}(x),
$$


for $P, P^{\prime} \in D, g, g^{\prime} \in K[x]_{\tau}$. Hence, putting $\Phi=\left(\right.$ id $\left.\otimes \overline{\phi_{A, \tau}}\right) \circ \Phi_{r}^{-1} \circ \Phi_{l}$, we have

$$
\begin{aligned}
W^{p, q} & \stackrel{\Phi}{\simeq} \bigoplus_{1 \leqq i_{1}<\cdots<i_{-q} \leqq d} \bigoplus_{\substack{\tau \succ \sigma \\
\operatorname{dim} \tau-\operatorname{dim} \sigma=p}} D \otimes_{K[x]} K[N A+Z(A \cap \tau)] e_{i_{1}} \wedge \cdots \wedge e_{i_{-q}} \\
& =\bigoplus_{\substack{1 \leqq i_{1}<\cdots<i_{-q} \leqq d\\
}} D \otimes_{K[x]}\left(L_{\sigma}^{p} \otimes_{K[x]} K[N A]\right) e_{i_{1}} \wedge \cdots \wedge e_{i_{-q}} \\
& =: \widetilde{W}^{p, q} .
\end{aligned}
$$

Next, we construct other differentials compatible with $\boldsymbol{W}^{\bullet}, \boldsymbol{\bullet}$. We define $\tilde{d}_{\mathrm{I}}$ and $\tilde{d}_{\mathrm{II}}(\boldsymbol{\beta})$ by

$$
\tilde{d}_{\mathrm{I}}^{p, q}=\mathrm{id} \otimes f^{p} \otimes \mathrm{id}: \widetilde{W}^{p, q} \rightarrow \widetilde{W}^{p+1, q},
$$

and

$$
\begin{aligned}
\tilde{d}_{\mathrm{II}}^{p, q}(\boldsymbol{\beta})\left(P \otimes \boldsymbol{t}^{\lambda} e_{i_{1}} \wedge \cdots \wedge e_{i_{-q}}\right) \\
\quad=\sum_{k=1}^{-q}(-1)^{r} P\left(\sum_{j=1}^{n} a_{i_{k} j} \partial_{j} x_{j}+\beta_{i_{k}}-\lambda_{i_{k}}\right) \otimes \boldsymbol{t}^{\lambda} e_{i_{1}} \wedge \cdots \wedge \hat{i_{k}} \wedge \cdots \wedge e_{i_{-q}},
\end{aligned}
$$

where

$$
P \in D, \lambda \in \bigcup_{\substack{\tau \succ \sigma \\ \operatorname{dim} \tau-\operatorname{dim} \sigma=p}}(N A+Z(A \cap \tau)), \quad i=1, \ldots, d .
$$

Then, we have the following.

PROPOSITION 3.2. We have the following.

(1) $\widetilde{\boldsymbol{W}}^{\bullet, \bullet}:=\left\{\widetilde{W}^{p, q}, \tilde{d}_{\mathrm{I}}^{p, q}, \tilde{d}_{\mathrm{II}}^{p, q}(\boldsymbol{\beta})\right\}$ is a double complex.

(2) $\boldsymbol{W}^{\bullet, \bullet} \stackrel{\Phi}{\simeq} \widetilde{\boldsymbol{W}}^{\bullet, \bullet}$ as double complexes.

PROOF. This clearly follows from the definitions of the complexes.

We examine the double complex $\widetilde{\boldsymbol{W}}^{\bullet \bullet \bullet}$ in detail. First, we have the following about $H_{\mathrm{I}}^{p}\left(\widetilde{\boldsymbol{W}}^{\bullet}, q\right)$.

Proposition 3.3. Assume that $A$ is Cohen-Macaulay. Then for all $q$ we have

(1) $H_{\mathrm{I}}^{p}\left(\widetilde{\boldsymbol{W}}^{\bullet}, q\right)=0\left(p \neq d_{\sigma}(=\operatorname{codim} \sigma)\right)$;

$$
H_{\mathrm{I}}^{d_{\sigma}}\left(\widetilde{\boldsymbol{W}}^{\bullet, q}\right) \simeq \bigoplus_{1 \leqq i_{1}<\cdots<i_{-q} \leqq d} D \otimes_{K[x]} \frac{K[\boldsymbol{Z} A]}{\sum_{\tau \succ \sigma, \text { facet }} K[\boldsymbol{N A}+\boldsymbol{Z}(A \cap \tau)]} e_{i_{1}} \wedge \cdots \wedge e_{i_{-q}} .
$$

PROOF. We recall that any localization of a Cohen-Macaulay ring is again a CohenMacaulay ring. Hence, it follows from Theorem 2.1 and [4, Section 6.3, Corollary 6.2.6], that

and

$$
H^{p}\left(\boldsymbol{L}_{\sigma}^{\bullet} \otimes_{K[x]} K[N A]\right)=0 \quad\left(p \neq d_{\sigma}\right),
$$

$$
H^{d_{\sigma}}\left(\boldsymbol{L}_{\sigma}^{\bullet} \otimes_{K[x]} K[\boldsymbol{N A} A) \simeq \frac{K[\boldsymbol{Z} A]}{\sum_{\tau \succ \sigma, \text { facet }} K[\boldsymbol{N A}+\boldsymbol{Z}(A \cap \tau)]} .\right.
$$


Since $D$ is flat over $K[x]$, we immediately obtain the statements.

Secondly, we have the following about $H_{\mathrm{II}}^{q}\left(\widetilde{\boldsymbol{W}}^{p, \bullet}\right)$.

Proposition 3.4. Assume that $A$ is Cohen-Macaulay. Then for all $p$ we have:

(1) $H_{\mathrm{II}}^{q}\left(\widetilde{\boldsymbol{W}}^{p, \bullet}\right)=0(q \neq 0)$;

(2) $H_{\mathrm{II}}^{0}\left(\widetilde{\boldsymbol{W}}^{p, \bullet}\right) \simeq L_{\sigma}^{p} \otimes_{K[x]} \mathcal{F} M_{A}(\boldsymbol{\beta})$.

Proof. This immediately follows from (10), Proposition 3.2, and the flatness of $K[x]_{\sigma}$ as a $K[x]$-module.

Note that, from the theory of spectral sequences, the following proposition holds.

Proposition 3.5. Let $\mathbf{W}^{\bullet \bullet \bullet}$ be a double complex. We assume that

$$
H_{\mathrm{I}}^{p}\left(\boldsymbol{W}^{\bullet}, q\right)=0
$$

for all $p, q$ with $p \neq p_{0}$, and that

$$
H_{\mathrm{II}}^{q}\left(\boldsymbol{W}^{p, \bullet}\right)=0
$$

for all $p, q$ with $q \neq q_{0}$. Then we have

$$
H_{\mathrm{I}}^{k}\left(H_{\mathrm{II}}^{q_{0}}\left(\widetilde{\boldsymbol{W}}^{\bullet \bullet \bullet}\right)\left[-q_{0}\right]\right) \simeq H_{\mathrm{II}}^{k}\left(H_{\mathrm{I}}^{p_{0}}\left(\widetilde{\boldsymbol{W}}^{\bullet, \bullet}\right)\left[-p_{0}\right]\right)
$$

for all $k$.

For a proof see, for instance, [11].

Finally, from these propositions, we obtain the following theorem.

THEOREM 3.6. Assume that A is Cohen-Macaulay. Then we have an isomorphism

$$
H^{k}\left(\boldsymbol{L}_{\sigma}^{\bullet} \otimes_{K[x]} \mathcal{F} M_{A}(\boldsymbol{\beta})\right) \simeq H_{\mathrm{II}}^{k}\left(H_{\mathrm{I}}^{d_{\sigma}}\left(\widetilde{\boldsymbol{W}}^{\bullet \bullet \bullet}\right)\left[-d_{\sigma}\right]\right)
$$

for all $k$.

Note that the complex

$$
H_{\mathrm{I}}^{d_{\sigma}}\left(\widetilde{\boldsymbol{W}}^{\bullet \bullet \bullet}\left[-d_{\sigma}\right]\right)
$$

is the Koszul complex associated with the module

$$
D \otimes_{K[x]} \frac{K[\boldsymbol{Z} A]}{\sum_{\tau \succ \sigma: \text { facet }} K[N A+\boldsymbol{Z}(A \cap \tau)]}
$$

and the morphisms induced by $\tilde{d}_{\mathrm{II}}^{d_{\sigma}, q}(\boldsymbol{\beta})$.

3.2. Deformation. In Section 3.1, we have constructed the Koszul complex (15). However, the computation of the cohomology of (15) is difficult. In this section, we give a nice decomposition as a $D$-module of the following module:

$$
N:=D \otimes_{K[x]} \frac{K[\boldsymbol{Z} A]}{\sum_{\tau \succ \sigma: \text { facet }} K[\boldsymbol{N A}+\boldsymbol{Z}(A \cap \tau)]} .
$$


In fact, each component of the decomposition is invariant under both the action of $D$ and the differential of the complex (15). Thus, this decomposition enables us to compute the cohomology of (15) more easily.

We easily think of a natural $\boldsymbol{Z} A$-grading decomposition for $N$ :

$$
N=\bigoplus_{\lambda \in P_{\sigma}} K[\partial]\left(1 \otimes t^{\lambda}\right),
$$

where

$$
P_{\sigma}=\boldsymbol{Z} A \backslash \bigcup_{\tau \succ \sigma: \text { facet }}(\boldsymbol{N} A+\boldsymbol{Z}(A \cap \tau)) .
$$

However, each component of the decomposition (16) is invariant under neither the action of $D$ nor the differential of the complex (15). Hence, we should decompose the module $N$ in another way. Now, for $\lambda \in P_{\sigma}$, put

$$
\eta^{\lambda}:=\sum_{\boldsymbol{v} \in N_{\sigma}} \frac{\partial^{v} x^{v}}{\boldsymbol{v} !}\left(1 \otimes \boldsymbol{t}^{\lambda}\right) \in D \otimes_{K[x]} \frac{K[\boldsymbol{Z} A]}{\sum_{\tau \succ \sigma: \text { facet }} K[\boldsymbol{N A}+\boldsymbol{Z}(A \cap \tau)]},
$$

where $\boldsymbol{N}_{\sigma}=\left\{\boldsymbol{v} \in \boldsymbol{N}^{n} \mid v_{j}=0\right.$ if $\left.j \in I_{\sigma}\right\}$. At first glance, the right-hand side of (18) simply looks like a formal sum. However, since for each $\lambda \in P_{\sigma}$ and $j \notin I_{\sigma}$ there exists $n_{j} \in N$ such that $\lambda+m \boldsymbol{a}_{j} \notin P_{\sigma}$ for all $m \geqq n_{j}$, and since $\lambda+A \boldsymbol{v} \notin P_{\sigma}$ implies that $\partial^{\boldsymbol{v}} \boldsymbol{x}^{\boldsymbol{v}} / \boldsymbol{v} !\left(1 \otimes \boldsymbol{t}^{\lambda}\right)=0$, the right-hand side of (18) is, in fact, a finite sum. Next we show some properties of $\eta^{\lambda}$ which help the following argument.

PROPOSITION 3.7. We have the following.

(1) If $j \notin I_{\sigma}$, then $x_{j} \eta^{\lambda}=0$. If $j \in I_{\sigma}$, then $x_{j} \eta^{\lambda}=\eta^{\lambda+a_{j}}$.

(2) Let $\rho^{(i)}(i=1, \ldots, d)$ be the endomorphism of $N$ defining the complex (15). Then

$$
\rho^{(i)}\left(\eta^{\lambda}\right)=\left(\sum_{j \in I_{\sigma}} a_{i j} \partial_{j} x_{j}+\beta_{i}-\lambda_{i}\right) \eta^{\lambda} .
$$

(3) The set $\left\{\eta^{\lambda} \mid \lambda \in P_{\sigma}\right\}$ is a basis of $N$ over $K$ [ग]; that is,

$$
N=\bigoplus_{\lambda \in P_{\sigma}} K[\partial] \eta^{\lambda} .
$$

Proof. (1) The statement is clear for $j \in I_{\sigma}$. Now assume that $j \notin I_{\sigma}$. Then,

$$
\begin{aligned}
& x_{j} \eta^{\lambda}=x_{j}\left(\sum_{\boldsymbol{v} \in N_{\sigma}} \frac{\partial^{v} x^{v}}{v !}\left(1 \otimes t^{\lambda}\right)\right) \\
& =\left(\sum_{v \in N_{\sigma}} \frac{x_{j} \partial^{v} x^{v}}{v !}\right)\left(1 \otimes t^{\lambda}\right) \\
& =\sum_{\boldsymbol{v} \in N_{\sigma}} \frac{\partial^{v} x^{\nu+\boldsymbol{e}_{j}}-v_{j} \partial^{\nu-\boldsymbol{e}_{j}} \boldsymbol{x}^{\boldsymbol{v}}}{\boldsymbol{v} !}\left(1 \otimes \boldsymbol{t}^{\lambda}\right)=0,
\end{aligned}
$$

where $\boldsymbol{e}_{j}:=\left(0, \ldots,{ }_{j}, \ldots, 0\right)$. 
(2) Recall the definition of $\rho^{(i)}$ (see (13)):

$$
\rho^{(i)}\left(\eta^{\lambda}\right)=\sum_{\boldsymbol{v} \in N_{\sigma}} \frac{\boldsymbol{\partial}^{\boldsymbol{v}} \boldsymbol{x}^{\boldsymbol{v}}}{\boldsymbol{v} !}\left(\sum_{j=1}^{n} a_{i j} \partial_{j} x_{j}+\beta_{i}-\lambda_{i}\right)\left(1 \otimes \boldsymbol{t}^{\lambda}\right) .
$$

If $j \notin I_{\sigma}$, then it follows that $\sum_{\boldsymbol{v} \in N_{\sigma}}\left(\partial^{v} x^{v} / v !\right) \partial_{j} x_{j}\left(1 \otimes \boldsymbol{t}^{\lambda}\right)=0$ as shown in 1 . Hence, we have

$$
\begin{aligned}
\rho^{(i)}\left(\boldsymbol{\eta}^{\lambda}\right) & =\sum_{\boldsymbol{v} \in N_{\sigma}} \frac{\partial^{v} \boldsymbol{x}^{\boldsymbol{v}}}{\boldsymbol{v} !}\left(\sum_{j=1}^{n} a_{i j} \partial_{j} x_{j}+\beta_{i}-\lambda_{i}\right)\left(1 \otimes \boldsymbol{t}^{\lambda}\right) \\
& =\sum_{\boldsymbol{v} \in \boldsymbol{N}_{\sigma}} \frac{\partial^{\boldsymbol{v}} \boldsymbol{x}^{\boldsymbol{v}}}{\boldsymbol{v} !}\left(\sum_{j \in I_{\sigma}} a_{i j} \partial_{j} x_{j}+\beta_{i}-\lambda_{i}\right)\left(1 \otimes \boldsymbol{t}^{\lambda}\right) \\
& =\left(\sum_{j \in I_{\sigma}} a_{i j} \partial_{j} x_{j}+\beta_{i}-\lambda_{i}\right)\left(\sum_{\boldsymbol{v} \in N_{\sigma}} \frac{\partial^{\boldsymbol{v}} \boldsymbol{x}^{\boldsymbol{v}}}{\boldsymbol{v} !}\left(1 \otimes \boldsymbol{t}^{\lambda}\right)\right) \\
& =\left(\sum_{j \in I_{\sigma}} a_{i j} \partial_{j} x_{j}+\beta_{i}-\lambda_{i}\right) \boldsymbol{\eta}^{\lambda} .
\end{aligned}
$$

(3) Put $\xi^{\lambda}=\sum_{v \in N_{\sigma}}\left(-\partial^{v} / v !\right) \eta^{\lambda+A v}$. Then $\xi^{\lambda}$ is also a finite sum. In fact,

$$
\begin{aligned}
\xi^{\lambda} & =\sum_{\mu \in N_{\sigma}} \frac{-\partial^{\mu}}{\mu !} \sum_{\nu \in N_{\sigma}} \frac{\partial^{v} x^{\nu}}{v !}\left(1 \otimes t^{\lambda+A \mu}\right) \\
& =\sum_{\mu, v \in N_{\sigma}} \frac{(-1)^{|\mu|} \partial^{\mu+v} x^{v+\mu}}{\mu ! v !} \otimes t^{\lambda}=1 \otimes t^{\lambda}
\end{aligned}
$$

Note that $\left\{1 \otimes \boldsymbol{t}^{\lambda} \mid \lambda \in P_{\sigma}\right\}$ is a basis of $N$ over $K[\partial]$ (see (16)). Hence, $\left\{\eta^{\lambda} \mid \lambda \in P_{\sigma}\right\}$ is also a basis of $N$.

We have obtained a new decomposition $N=\bigoplus_{\lambda \in P_{\sigma}} K[\partial] \eta^{\lambda}$. However, each component does not have a $D$-module structure. Considering an equivalence relation on $P_{\sigma}$, we resolve this problem. Note that, for any $\tau \succ \sigma, N A+\boldsymbol{Z}(A \cap \tau)$ contains $\boldsymbol{Z}(A \cap \sigma)$. Hence, we may define a relation $\sim$ on $P_{\sigma}$ by

$$
\lambda \sim \lambda^{\prime} \Leftrightarrow \lambda-\lambda^{\prime} \in Z(A \cap \sigma) .
$$

It is clear that this relation $\sim$ is an equivalence relation. We denote the quotient by $\tilde{E}_{\sigma}$, and for $\Lambda \in \tilde{E}_{\sigma}$ we set

$$
N_{\Lambda}:=\bigoplus_{\lambda \in \Lambda} K[\partial] \eta^{\lambda}
$$

Then, from Proposition 3.7, we can easily show that $N_{\Lambda}$ has a $D$-module structure by multiplying an element of $D$ from the left. Hence, we can give $N$ a new decomposition as a $D$-module:

$$
N=\bigoplus_{\Lambda \in \tilde{E}_{\sigma}} N_{\Lambda}
$$


We note that, by Proposition 3.7 again, $N_{\Lambda}$ is invariant under the morphism $\rho$. However, we are not able to give such a decomposition to $D \otimes_{K[x]} K[N A+Z(A \cap \sigma)]$.

To simplify the description, we denote $K\left\langle x_{j}, \partial_{j} \mid j \in I_{\sigma}\right\rangle$ and $K\left\langle x_{j}, \partial_{j} \mid j \notin I_{\sigma}\right\rangle$ by $D_{(\sigma)}$ and $D_{\left(\sigma^{c}\right)}$, respectively. Similarly, we denote $K\left[x_{j} \mid j \in I_{\sigma}\right]$ and $K\left[x_{j} \mid j \notin I_{\sigma}\right]$ by $K\left[\boldsymbol{x}_{\sigma}\right]$ and $K\left[\boldsymbol{x}_{\sigma^{c}}\right]$, respectively. Recall that we can identify $D$ (resp. $K[\boldsymbol{x}]$ ) with the external product $D_{\left(\sigma^{c}\right)} \bigotimes_{K} D_{(\sigma)}$ (resp. $\left.K\left[\boldsymbol{x}_{\sigma^{c}}\right] \bigotimes_{K} K\left[\boldsymbol{x}_{\sigma}\right]\right)$. Under these identifications, we obtain the following proposition.

Proposition 3.8. Let $\Lambda \in \tilde{E}_{\sigma}$ and fix $\lambda_{0} \in \Lambda$. Then there exists a D-isomorphism

$$
\Phi_{\lambda_{0}}: N_{\Lambda} \stackrel{\sim}{\rightarrow} \frac{D_{\left(\sigma^{c}\right)}}{D_{\left(\sigma^{c}\right)}\left\langle\boldsymbol{x}_{\left.\sigma^{c}\right\rangle}\right.} \otimes_{K} D_{(\sigma)} \otimes_{K\left[\boldsymbol{x}_{\sigma}\right]} K[\boldsymbol{Z}(A \cap \sigma)] .
$$

PRoOF. Note that $N_{\Lambda}=\bigoplus_{\lambda^{\prime} \in \boldsymbol{Z}(A \cap \sigma)} K[\partial] \eta^{\lambda^{\prime}+\lambda_{0}}$. First we define a $K[\partial]$-morphism

$$
\Phi_{\lambda_{0}}: N_{\Lambda} \rightarrow \frac{D_{\left(\sigma^{c}\right)}}{D_{\left(\sigma^{c}\right)}\left\langle\boldsymbol{x}_{\sigma^{c}}\right\rangle} \otimes_{K} D_{(\sigma)} \otimes_{K\left[\boldsymbol{x}_{\sigma}\right]} K[\boldsymbol{Z}(A \cap \sigma)]
$$

by $\Phi_{\lambda_{0}}\left(\eta^{\lambda^{\prime}+\lambda_{0}}\right)=\overline{1} \otimes 1 \otimes t^{\lambda^{\prime}}$. Secondly, we consider the morphism

$$
\frac{D_{\left(\sigma^{c}\right)}}{D_{\left(\sigma^{c}\right)}\left\langle\boldsymbol{x}_{\sigma^{c}}\right\rangle} \times D_{(\sigma)} \times K[\boldsymbol{Z}(A \cap \sigma)] \rightarrow N_{\Lambda},
$$

$\left(\bar{P}, Q, \boldsymbol{t}^{\lambda^{\prime}}\right) \mapsto P Q \eta^{\lambda^{\prime}+\lambda_{0}}\left(P \in D_{\left(\sigma^{c}\right)}, Q \in D_{(\sigma)}, \lambda^{\prime} \in \boldsymbol{Z}(A \cap \sigma)\right)$. It clearly follows from Proposition 3.7(1) that this is well defined and balanced. Hence, from the universal mapping property, we have a morphism

$$
\Psi_{\lambda_{0}}: \frac{D_{\left(\sigma^{c}\right)}}{D_{\left(\sigma^{c}\right)}\left\langle\boldsymbol{x}_{\sigma^{c}}\right\rangle} \otimes_{K} D_{(\sigma)} \otimes_{K\left[x_{\sigma}\right]} K[\boldsymbol{Z}(A \cap \sigma)] \rightarrow N_{\Lambda}
$$

with the equality $\Psi_{\lambda_{0}}\left(\bar{P} \otimes Q \otimes \boldsymbol{t}^{\lambda^{\prime}}\right)=P Q \eta^{\lambda^{\prime}+\lambda_{0}}$. We easily see that $\Psi_{\lambda_{0}}$ is a $D$-morphism, $\Psi_{\lambda_{0}} \circ \Phi_{\lambda_{0}}=$ id and $\Phi_{\lambda_{0}} \circ \Psi_{\lambda_{0}}=$ id.

Now we consider the $D_{(\sigma)}$-endomorphism

$$
\tilde{\rho}_{\lambda_{0}}^{(i)}: D_{(\sigma)} \otimes_{K\left[x_{\sigma}\right]} K[\boldsymbol{Z}(A \cap \sigma)] \rightarrow D_{(\sigma)} \otimes_{K\left[\boldsymbol{x}_{\sigma}\right]} K[\boldsymbol{Z}(A \cap \sigma)],
$$

$\tilde{\rho}_{\lambda_{0}}^{(i)}\left(1 \otimes \boldsymbol{t}^{\lambda^{\prime}}\right)=\left(\sum_{j \in I_{\sigma}} a_{i j} \partial_{j} x_{j}+\beta_{i}-\lambda_{i}^{\prime}-\lambda_{0, i}\right) \otimes \boldsymbol{t}^{\lambda^{\prime}}$. Then it is clear that $\Phi_{\lambda_{0}} \circ \rho^{(i)}=$ (id $\left.\otimes \tilde{\rho}_{\lambda_{0}}^{(i)}\right) \circ \Phi_{\lambda_{0}}$, so we define the Koszul complex $\widetilde{\boldsymbol{K}}^{\bullet}:=\operatorname{Kos}^{\bullet}\left(D_{(\sigma)} \otimes K[\boldsymbol{Z}(A \cap \sigma)] ; \tilde{\rho}_{\lambda}^{(i)}\right)$ associated with $D_{(\sigma)} \otimes K[Z(A \cap \sigma)]$ and $\left\{\tilde{\rho}_{\lambda}^{(i)} \mid i=1, \ldots, d\right\}$ as in Section 3.1. By Corollary 2.2, Theorem 3.6, Proposition 3.7(3) and Proposition 3.8, we deduce the following theorem.

THEOREM 3.9. Assume that $A$ is Cohen-Macaulay and fix a system of representatives $\{\lambda\}$ of $\tilde{E}_{\sigma}$. Then we have

$$
H_{\left[X_{\sigma}\right]}^{k}\left(\mathcal{F} M_{A}(\boldsymbol{\beta})\right) \simeq \bigoplus_{\lambda} \frac{D_{\left(\sigma^{c}\right)}}{D_{\left(\sigma^{c}\right)}\left\langle\boldsymbol{x}_{\sigma^{c}}\right\rangle} \otimes H^{k-d_{\sigma}}\left(\operatorname{Kos}^{\bullet}\left(D_{(\sigma)} \otimes K[\boldsymbol{Z}(A \cap \sigma)] ; \tilde{\rho}_{\lambda}^{(i)}\right) .\right.
$$


3.3. Main theorem. Using (23), we explicitly compute $H_{\left[X_{\sigma}\right]}^{k}\left(\mathcal{F} M_{A}(\boldsymbol{\beta})\right)$. It is sufficient to compute the Koszul complex $\widetilde{\boldsymbol{K}}^{\bullet}$ for each $\lambda$. Note that $r_{\sigma}:=\operatorname{rank}_{K}(A \cap \sigma) \leqq$ $\operatorname{rank}_{K}([A \cap \sigma ; \boldsymbol{\beta}-\lambda])$, where $[A \cap \sigma ; \boldsymbol{\beta}-\lambda]$ means the enlarged coefficient matrix of $A \cap \sigma$ with $\beta-\lambda$. In fact, the vanishing of the cohomology completely depends on whether $r_{\sigma}$ is equal to $\operatorname{rank}_{K}([A \cap \sigma ; \boldsymbol{\beta}-\lambda])$ or not. We consider two cases. Let $V$ be the subspace $\left\langle\tilde{\rho}_{\lambda}^{(i)} \mid i=1, \ldots, d\right\rangle_{K}$ of $\operatorname{End}_{K}\left(D_{(\sigma)} \otimes K[Z(A \cap \sigma)]\right)$.

First we consider the case $r_{\sigma} \neq \operatorname{rank}_{K}(A \cap \sigma ; \boldsymbol{\beta})$. In this case, we easily see that the identity morphism 1 belongs to $V$. Then we obtain the following proposition.

Proposition 3.10. Let $M$ be a $K$-vector space, $\left\{f_{i}\right\}_{i=1}^{d}$ a sequence in $\operatorname{End}_{K}(M)$ and $\operatorname{Kos}^{\bullet}\left(M ; f_{i}, i=1, \ldots, d\right)$ the Koszul complex associated to $M$ and $\left\{f_{i}\right\}_{i=1}^{d}$. If the identity $1_{M}$ is contained in the linear span of $\left\{f_{i}\right\}_{i=1}^{d}$, then we have

$$
H^{k}\left(\operatorname{Kos}^{\bullet}\left(M ; f_{i}, i=1, \ldots, d\right)\right)=0
$$

for all $k$.

Proof. The proof is similar to that of [4, Theorem 1.6.17]. Note that $M=1_{M}(M)$ and that the proof works even in this case.

From this proposition, in the case $r_{\sigma} \neq \operatorname{rank}_{K}(A \cap \sigma ; \boldsymbol{\beta})$, we have

$$
H^{k}\left(\operatorname{Kos}^{\bullet}\left(D_{(\sigma)} \otimes K[Z(A \cap \sigma)] ; \tilde{\rho}_{\lambda}^{(i)}\right)\right)=0
$$

for all $k$.

Secondly, we consider the case $r_{\sigma}=\operatorname{rank}_{K}(A \cap \sigma ; \boldsymbol{\beta}-\lambda)$. In this case, note that $r_{\sigma}$ generators among $\left\{\tilde{\rho}_{\lambda}^{(i)} \mid i=1, \ldots, d\right\}$ span $V$. Without loss of generality, we may assume that $\tilde{\rho}_{\lambda}^{(1)}, \ldots, \tilde{\rho}_{\lambda}^{\left(r_{\sigma}\right)}$ span $V$. Then $\tilde{\rho}_{\lambda}^{\left(r_{\sigma}+1\right)}, \ldots, \tilde{\rho}_{\lambda}^{(d)} \in V$. Note that we have the following.

Proposition 3.11. Let $M$ be a $K$-vector space and $\left\{f_{i}\right\}_{i=1}^{d+d^{\prime}}$ a sequence in $\operatorname{End}_{K}(M)$. If $f_{d+1}, \ldots, f_{d+d^{\prime}} \in\left\langle f_{i} \mid i=1, \ldots, d\right\rangle_{K}$, then we have

$$
H^{k-d^{\prime}}\left(\operatorname{Kos}^{\bullet}\left(M ; f_{i}, i=1, \ldots, d+d^{\prime}\right)\right) \simeq H^{k}\left(\operatorname{Kos}^{\bullet}\left(M ; f_{i}, i=1, \ldots, d\right)\right)^{\oplus\left(\begin{array}{c}
d^{\prime} \\
d^{\prime}-k
\end{array}\right)}
$$

for all $k$.

The proof is similar to that of [4, Proposition 1.6.21].

From this proposition and the acyclicity of the Koszul complex in the case of $\sigma=Q_{\geq 0} A$ (see Proposition 3.4), we immediately have

$$
\begin{aligned}
& H^{k-d_{\sigma}}\left(\operatorname{Kos}^{\bullet}\left(D_{(\sigma)} \otimes K[Z(A \cap \sigma)] ; \tilde{\rho}_{\lambda}^{(i)} i=1, \ldots, d\right)\right.
\end{aligned}
$$

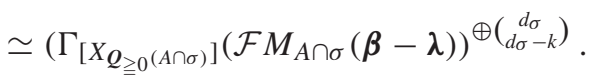

(Recall that $d=r_{\sigma}+d_{\sigma}$.) Note that, if $\lambda \simeq \lambda^{\prime}$, then

$$
\begin{aligned}
& \operatorname{rank}_{K}(A \cap \sigma ; \boldsymbol{\beta}-\lambda)=\operatorname{rank}_{K}\left(A \cap \sigma ; \boldsymbol{\beta}-\boldsymbol{\lambda}^{\prime}\right), \\
& \Gamma_{\left[X_{\boldsymbol{Q}_{\geqq 0}(A \cap \sigma)}\left(\mathcal{F} M_{A \cap \sigma}(\boldsymbol{\beta}-\lambda)\right)\right.} \simeq \Gamma_{\left[X_{\left.\boldsymbol{Q}_{\geqq 0}(A \cap \sigma)\right]}\left(\mathcal{F} M_{A \cap \sigma}\left(\boldsymbol{\beta}-\boldsymbol{\lambda}^{\prime}\right)\right) .\right.}
\end{aligned}
$$


Thus, in both cases, these results are independent of the choice of representatives for $\Lambda$ up to

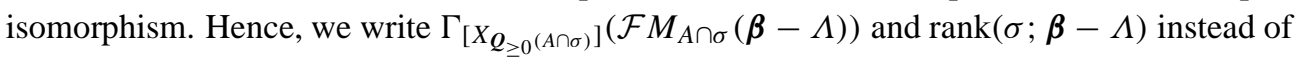
$\Gamma_{\left[X_{\boldsymbol{Q}_{>0}(A \cap \sigma)}\right.}\left(\mathcal{F} M_{A \cap \sigma}(\boldsymbol{\beta}-\lambda)\right)$ and $\operatorname{rank}_{K}(A \cap \sigma ; \boldsymbol{\beta}-\lambda)$, respectively. Putting $\tilde{E}_{\sigma}(\boldsymbol{\beta}):=\{\Lambda \mid$ $\left.\operatorname{rank}(\sigma ; \boldsymbol{\beta}-\Lambda)=r_{\sigma}\right\}$, we finally obtain the main theorem by applying Theorem 3.9 to them.

THEOREM 3.12 (Main theorem). Assume that $A$ is Cohen-Macaulay. For all $k$ and all $\sigma \in \mathcal{S}_{A}$, we have

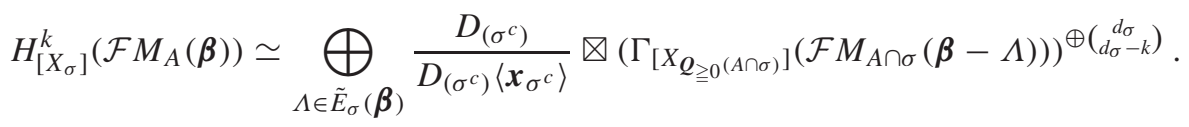

In particular, $\tilde{E}_{\sigma}(\boldsymbol{\beta})=\varnothing$ if and only if $\mathrm{R} \Gamma_{\left[X_{\sigma}\right]}\left(\mathcal{F} M_{A}(\boldsymbol{\beta})\right)=0$.

4. Examples and an application of the main theorem. Throughout this section, we assume $K$ to be the complex number field $\boldsymbol{C}$.

4.1. Some examples of Cohen-Macaulay type. We provide some examples of Cohen-Macaulay type for computation of the algebraic local cohomology modules.

EXAMPLE 4.1. Let $d=1$ and $A_{1}=(1)$. Then we easily see the following:

$$
\boldsymbol{Q}_{\geq 0} A=\{p \in \boldsymbol{Q} \mid p \geq 0\}, \quad \mathcal{S}_{A}=\left\{\boldsymbol{Q}_{\geq 0},\{0\}\right\}, \quad I_{A}(x)=0, \quad \mathrm{~V}\left(I_{A}(x)\right)=\boldsymbol{C} .
$$

Note that the orbits $X_{\sigma}$ corresponding to $\sigma \in \mathcal{S}_{A}$ are given by

$$
X_{\{0\}}=\{0\}, \quad X_{\boldsymbol{Q}_{\geq 0}}=\boldsymbol{C}^{\times}=\{x \in \boldsymbol{C} \mid x \neq 0\} .
$$

We examine $\tilde{E}_{\sigma}(\boldsymbol{\beta})$ corresponding to $\sigma \in \mathcal{S}_{A}$. First, for $\sigma=\{0\}$, we have

$$
P_{\{0\}}=\boldsymbol{Z} \backslash N, \quad \tilde{E}_{\{0\}}=\boldsymbol{Z} \backslash N .
$$

Note that $r_{\{0\}}$ is equal to 0 . Hence, we have

$$
\tilde{E}_{\{0\}}(\beta)= \begin{cases}\{\beta\} & \beta \in \boldsymbol{Z} \backslash N \\ \varnothing & \text { otherwise }\end{cases}
$$

Secondly, for $\sigma=Q_{\geq 0}$, we have

$$
P_{Q_{\geq 0}}=\boldsymbol{Z}, \quad \tilde{E}_{\boldsymbol{Q}_{\geq 0}}=\{0\} .
$$

Note that $r_{Q_{\geq 0}}$ is equal to $d$. Hence, we have $\tilde{E}_{\boldsymbol{Q}_{\geq 0}}(\beta)=\{0\}$ for an arbitrary parameter $\beta$.

Table 1 summarizes the results of the calculation. The first row of the table means the elements of $\mathcal{S}_{A}$. The second row 'Orbits $X_{(\cdot)}$ ' means the orbit $X_{\sigma}$ corresponding to $\sigma \in \mathcal{S}_{A}$. The third row $\tilde{E}_{(\cdot)} \neq \varnothing$ means the condition of $\boldsymbol{\beta}$ for non-emptiness of $\tilde{E}_{\sigma}(\boldsymbol{\beta})$ corresponding to $\sigma \in \mathcal{S}_{A}$.

EXAmPLE 4.2. $A_{2}=\left(\begin{array}{lll}1 & 1 & 1 \\ 0 & 1 & 2\end{array}\right)$. See Table 2. Note that

$$
\begin{gathered}
\left|\tilde{E}_{\boldsymbol{Q}_{\geq 0}{ }^{\mathrm{t}}(1,0)}(\boldsymbol{\beta})\right|=1 \Leftrightarrow \beta_{2}=-1,-2, \ldots, \\
\left|\tilde{E}_{\boldsymbol{Q}_{\geq 0}{ }^{\mathrm{t}}(1,2)}(\boldsymbol{\beta})\right|=1 \Leftrightarrow 2 \beta_{1}-\beta_{2}=-1,-2, \ldots, \\
\left|\tilde{E}_{\{0\}}(\boldsymbol{\beta})\right|=1 \Leftrightarrow 2 \beta_{1}-\beta_{2}, \beta_{2}=-1,-2, \ldots .
\end{gathered}
$$


TABle $1 . \quad A_{1}$.

\begin{tabular}{ccc}
\hline $\mathcal{S}_{A}$ & $\{0\}$ & $\boldsymbol{Q}_{\geqq 0}$ \\
\hline Orbits $X_{(\cdot)}$ & $\{0\}$ & $\{x \neq 0\}$ \\
$\tilde{E}_{(\cdot)} \neq \varnothing$ & $\beta \in \boldsymbol{Z} \backslash \boldsymbol{N}$ & Arbitrary \\
\hline
\end{tabular}

TABle 2. $A_{2}$.

\begin{tabular}{|c|c|c|}
\hline $\mathcal{S}_{A}$ & $\{0\}$ & $\boldsymbol{Q}_{\geqq 0}\left(\begin{array}{l}1 \\
0\end{array}\right)$ \\
\hline Orbits $X_{(\cdot)}$ & $\{0\}$ & $\left\{x_{1} \neq 0, x_{2}=x_{3}=0\right\}$ \\
\hline$\tilde{E}_{(\cdot)} \neq \varnothing$ & $2 \beta_{1}-\beta_{2}, \beta_{2} \in \boldsymbol{Z} \backslash \boldsymbol{N}$ & $\beta_{2} \in \boldsymbol{Z} \backslash \boldsymbol{N}$ \\
\hline $\mathcal{S}_{A}$ & $Q_{\geqq 0}\left(\begin{array}{l}1 \\
2\end{array}\right)$ & $Q_{\geqq 0} A$ \\
\hline Orbits $X_{(\cdot)}$ & $\left\{x_{1}=x_{2}=0, x_{3} \neq 0\right\}$ & $\left\{x_{2}^{2}-x_{1} x_{3}=0, x_{1} x_{2} x_{3} \neq 0\right\}$ \\
\hline$\tilde{E}_{(\cdot)} \neq \varnothing$ & $2 \beta_{1}-\beta_{2} \in \boldsymbol{Z} \backslash \boldsymbol{N}$ & Arbitrary \\
\hline $\mathcal{S}_{A}$ & $\{0\}$ & $Q_{\geqq 0}\left(\begin{array}{l}2 \\
0\end{array}\right)$ \\
\hline Orbits $X_{(\cdot)}$ & $\{0\}$ & $\left\{x_{1} \neq 0, x_{2}=x_{3}=0\right\}$ \\
\hline$\tilde{E}_{(\cdot)} \neq \varnothing$ & $\beta_{1}, \beta_{2} \in \boldsymbol{Z} \backslash \boldsymbol{N}$ & $\beta_{2} \in \boldsymbol{Z} \backslash N_{>0}$ \\
\hline $\mathcal{S}_{A}$ & $Q_{\geqq 0}\left(\begin{array}{l}0 \\
1\end{array}\right)$ & $Q_{\geqq 0} A$ \\
\hline Orbits $X_{(\cdot)}$ & $\left\{x_{1}=x_{2}=0, x_{3} \neq 0\right\}$ & $\left\{x_{2}^{2}-x_{1} x_{3}^{2}=0, x_{1} x_{2} x_{3} \neq 0\right\}$ \\
\hline$\tilde{E}_{(\cdot)} \neq \varnothing$ & $\beta_{1} \in \boldsymbol{Z} \backslash N$ & Arbitrary \\
\hline
\end{tabular}

EXAmPLE 4.3. $A_{3}=\left(\begin{array}{lll}2 & 1 & 0 \\ 0 & 1 & 1\end{array}\right)$ : not homogeneous. See Table 3. Note that

$$
\begin{gathered}
\left|\tilde{E}_{\boldsymbol{Q}_{\geqq 0}{ }^{\mathrm{t}}(2,0)}(\boldsymbol{\beta})\right|=1 \Leftrightarrow \beta_{2}=0, \\
\left|\tilde{E}_{\boldsymbol{Q}_{\geqq 0}{ }^{\mathrm{t}(2,0)}}(\boldsymbol{\beta})\right|=2 \Leftrightarrow \beta_{2}=-1,-2, \ldots, \\
\left|\tilde{E}_{\boldsymbol{Q}_{\geqq 0}{ }^{\mathrm{t}}(0,1)}(\boldsymbol{\beta})\right|=1 \Leftrightarrow \beta_{1}=-1,-2, \ldots, \\
\left|\tilde{E}_{\{0\}}(\boldsymbol{\beta})\right|=2 \Leftrightarrow \beta_{1}, \beta_{2}=-1,-2, \ldots .
\end{gathered}
$$

EXAmple 4.4. $A_{4}=\left(\begin{array}{cccc}1 & 0 & 0 & 0 \\ 0 & 1 & 0 & 1 \\ 0 & 0 & 1 & -1\end{array}\right)$. Put $a_{1}={ }^{\mathrm{t}}(1,0,0), a_{2}={ }^{\mathrm{t}}(0,1,0), a_{3}={ }^{\mathrm{t}}(0,0,1)$, $a_{4}={ }^{\mathrm{t}}(0,1,-1)$. See Table 4.

Note that for any $\sigma \in \mathcal{S}_{A}, \tilde{E}_{(\sigma)} \neq \varnothing$ implies $\left|\tilde{E}_{\sigma}(\boldsymbol{\beta})\right|=1$. 
TABLE $4 . A_{4}$.

\begin{tabular}{|c|c|c|}
\hline $\mathcal{S}_{A}$ & $\{0\}$ & $\boldsymbol{Q}_{\geqq 0} a_{1}$ \\
\hline Orbits $X_{(\cdot)}$ & $\{0\}$ & $\left\{x_{1} \neq 0, x_{2}=x_{3}=x_{4}=0\right\}$ \\
\hline$\tilde{E}_{(\cdot)} \neq \varnothing$ & $\beta_{1}, \beta_{2}, \beta_{1}+\beta_{2}, \beta_{2}+\beta_{3} \in \boldsymbol{Z} \backslash \boldsymbol{N}$ & $\beta_{2}, \beta_{2}+\beta_{3} \in \boldsymbol{Z} \backslash \boldsymbol{N}$ \\
\hline $\mathcal{S}_{A}$ & $Q_{\geqq 0} a_{2}$ & $Q_{\geqq 0} a_{3}$ \\
\hline Orbits $X_{(\cdot)}$ & $\left\{x_{2} \neq 0, x_{1}=x_{3}=x_{4}=0\right\}$ & $\left\{x_{3} \neq 0, x_{1}=x_{2}=x_{4}=0\right\}$ \\
\hline$\tilde{E}_{(\cdot)} \neq \varnothing$ & $\beta_{1}, \beta_{1}+\beta_{3} \in \boldsymbol{Z} \backslash \boldsymbol{N}$ & $\beta_{1}, \beta_{2} \in \boldsymbol{Z} \backslash N$ \\
\hline $\mathcal{S}_{A}$ & $Q_{\geqq 0} a_{4}$ & $Q_{\geqq 0}\left(a_{1}, a_{3}\right)$ \\
\hline Orbits $X_{(\cdot)}$ & $\left\{x_{4} \neq 0, x_{1}=x_{2}=x_{3}=0\right\}$ & $\left\{x_{1} x_{3} \neq 0, x_{2}=x_{4}=0\right\}$ \\
\hline$\tilde{E}_{(\cdot)} \neq \varnothing$ & $\beta_{1}+\beta_{3}, \beta_{2}+\beta_{3} \in Z \backslash \boldsymbol{N}$ & $\beta_{2} \in \boldsymbol{Z} \backslash \boldsymbol{N}$ \\
\hline $\mathcal{S}_{A}$ & $Q_{\geqq 0}\left(a_{2}, a_{3}\right)$ & $Q_{\geqq 0}\left(a_{2}, a_{4}\right)$ \\
\hline Orbits $X_{(\cdot)}$ & $\left\{x_{2} x_{3} \neq 0, x_{1}=x_{4}=0\right\}$ & $\left\{x_{2} x_{4} \neq 0, x_{1}=x_{3}=0\right\}$ \\
\hline$\tilde{E}_{(\cdot)} \neq \varnothing$ & $\beta_{1} \in \boldsymbol{Z} \backslash N$ & $\beta_{1}+\beta_{3} \in \boldsymbol{Z} \backslash \boldsymbol{N}$ \\
\hline $\mathcal{S}_{A}$ & $Q_{\geqq 0}\left(a_{1}, a_{4}\right)$ & $\boldsymbol{Q}_{\geqq 0} A$ \\
\hline Orbits $X_{(\cdot)}$ & $\left\{x_{1} x_{4} \neq 0, x_{2}=x_{3}=0\right\}$ & $\left\{x_{1} x_{2} x_{3} x_{4} \neq 0, x_{1} x_{2}-x_{3} x_{4}=0\right\}$ \\
\hline$\tilde{E}_{(\cdot)} \neq \varnothing$ & $\beta_{2}+\beta_{3} \in \boldsymbol{Z} \backslash \boldsymbol{N}$ & Arbitrary \\
\hline
\end{tabular}

4.2. The solution sheaf of $\mathcal{F} M_{A}(\boldsymbol{\beta})$. In this Section, we examine the solution sheaf of $\mathcal{F} M_{A}(\boldsymbol{\beta})$. Note that Hotta and Kashiwara gave in [9] the following relation between the solution sheaf of a system and that of its Fourier transform.

THEOREM 4.5 (Hotta-Kashiwara [9, Theorem 3.2]). Let M be a coherent homogeneous D-module (see Section 2.1), and $Z$ the closed subset of $\boldsymbol{C}_{x}^{n} \times \boldsymbol{C}_{y}^{n}$ given by

$$
\left\{(x, y) \in \boldsymbol{C}_{x}^{n} \times \boldsymbol{C}_{y}^{n} \mid \operatorname{Re}\langle x, y\rangle \geqq 0\right\} .
$$

Let $\tau$ and $\pi$ be the projections from $\boldsymbol{C}_{x}^{n} \times \boldsymbol{C}_{y}^{n}$ onto $\boldsymbol{C}_{x}^{n}$ and $\boldsymbol{C}_{y}^{n}$, respectively. Then we have

$$
\operatorname{Sol}(\mathcal{F} M) \simeq \mathrm{R} \pi_{*} \mathrm{R} \Gamma_{Z}\left(\tau^{-1} \operatorname{Sol}(M)\right)
$$

in the analytic category.

Recall that, if there exists an integral vector $\boldsymbol{c} \in \boldsymbol{Z}^{d}$ such that

$$
\boldsymbol{c} A={ }^{\mathrm{t}}(1,1, \ldots, 1), \quad \text { homogeneity condition , }
$$


then $M_{A}(\boldsymbol{\beta})$ and $\mathcal{F} M_{A}(\boldsymbol{\beta})$ are homogeneous regular holonomic $D$-modules (see $\left.[8,9]\right)$. We assume the condition (27). Then, by applying Theorem 4.5 to $\mathcal{F} M_{A}(\boldsymbol{\beta})$, we easily deduce that

$$
\operatorname{Sol}\left(\mathcal{F} M_{A}(\boldsymbol{\beta})\right) \simeq \mathrm{R} \pi_{*} \mathrm{R} \Gamma_{Z}\left(\tau^{-1} \operatorname{Sol}\left(M_{A}(\boldsymbol{\beta})\right)\right) .
$$

Furthermore, Gel'fand et al. [7] proved the following theorem.

THEOREM 4.6 (Gel'fand et al. [7]). We have

$$
\mathrm{Ch}\left(\mathcal{F} M_{A}(\boldsymbol{\beta})\right) \subset \coprod_{\sigma \in \mathcal{S}(A)} T_{X_{\sigma}}^{*}\left(\boldsymbol{C}^{n}\right)
$$

where $\mathrm{Ch}$ means the characteristic variety and $T_{X_{\sigma}}^{*}\left(C^{n}\right)$ means the conormal bundle of $X_{\sigma} \subset$ $\boldsymbol{C}^{n}$.

Note that this statement is equivalent to the statement that $\operatorname{Sol}\left(\mathcal{F} M_{A}(\boldsymbol{\beta})\right)$ is constructible with respect to the algebraic stratification $\left\{X_{\sigma} \mid \sigma \in \mathcal{S}_{A}\right\}$.

Let $\iota_{\sigma}: X_{\sigma} \rightarrow C^{n}$ be the inclusion map of smooth algebraic varieties, and $\iota_{\sigma}^{\text {an }}$ its analytification. Then, note that for a regular holonomic $D$-module $M$, we have

$$
\iota_{\sigma}^{-1} \operatorname{Sol}(M) \simeq \operatorname{Sol}\left(\iota_{\sigma}^{\dagger} M\right)
$$

(see, for instance, [10] and [3]). Here, $\iota_{\sigma}^{\dagger}$ means the inverse image functor with respect to $\iota_{\sigma}^{\text {an }}$, (the sign 'an' is omitted for brevity). Now recall that we can identify $X_{\sigma}$ with the algebraic torus $T_{\sigma}:=\left(\boldsymbol{C}^{\times}\right)^{\operatorname{dim} \sigma}$ (see (4)). If $\sigma=\boldsymbol{Q}_{\geq 0} A$, then from Kashiwara's equivalence we easily see that $\iota_{\sigma}^{\dagger} \mathcal{F} M_{A}(\boldsymbol{\beta})$ is isomorphic to a connection of rank one $\mathcal{R}(\sigma ; \boldsymbol{\beta}):=D_{T_{\sigma}} / D_{T_{\sigma}}\left\langle t_{i} \partial_{t_{i}}\right.$ $\bar{\beta}_{i}\left|i=1, \ldots, r_{\sigma}\right\rangle$ (see [6, Sections 3.1 and 4.2]), where $\overline{\boldsymbol{\beta}}:=-\sum_{j \in I_{\sigma}} \boldsymbol{a}_{j}-\boldsymbol{\beta}$. For the other faces, applying Kashiwara's equivalence to Theorem 3.12, we have the following.

COROllary 4.7. Assume that A is Cohen-Macaulay with the condition (27). Then we have

$$
H^{k} \iota_{\sigma}^{\dagger} \mathcal{F} M_{A}(\boldsymbol{\beta}) \simeq \bigoplus_{\Lambda \in \tilde{E}_{\sigma}(\boldsymbol{\beta})}(\mathcal{R}(\sigma ; \boldsymbol{\beta}-\Lambda))^{\oplus\left(\begin{array}{c}
d_{\sigma} \\
d_{\sigma}-k
\end{array}\right)}
$$

for all $k$ and all $\sigma \in \mathcal{S}_{A}$.

Note that, for a connection $L, \operatorname{Sol}(L)$ has no higher cohomology. So we define $\mathcal{L}(\sigma ; \bullet)=$ $H^{0} \operatorname{Sol}(\mathcal{R}(\sigma ; \bullet))$. Then we have the following theorem.

THEOREM 4.8. Assume that A is Cohen-Macaulay with the condition (27). Then we have

$$
\iota_{\sigma}^{-1}\left(H^{k} \operatorname{Sol}\left(\mathcal{F} M_{A}(\boldsymbol{\beta})\right)\right) \simeq \bigoplus_{\Lambda \in \tilde{E}_{\sigma}(\boldsymbol{\beta})}(\mathcal{L}(\sigma ; \boldsymbol{\beta}-\Lambda))^{\oplus\left(\left(_{d_{\sigma}-k}^{d_{\sigma}}\right)\right.}
$$

for all $k$ and all $\sigma \in \mathcal{S}_{A}$.

Proof. Fix $\sigma \in \mathcal{S}_{A}$, and take the solutions of both sides in (30). Then we immediately deduce the theorem by (29). 
We see that, when $A$ is Cohen-Macaulay, the structure of the local cohomology modules $H_{\left[X_{\sigma}\right]}^{k}\left(\mathcal{F} M_{A}(\boldsymbol{\beta})\right)$ (resp. the constructible sheaf $\operatorname{Sol}\left(\mathcal{F} M_{A}(\boldsymbol{\beta})\right)$ ) has symmetry with respect to the cardinality of the direct summand. Moreover, the structure is completely controlled by $\tilde{E}_{\sigma}(\boldsymbol{\beta})$. In this sense, we can identify $\tilde{E}_{\sigma}(\boldsymbol{\beta})$ with the restriction of the solution sheaf to the orbit $X_{\sigma}$. However, when $A$ is not Cohen-Macaulay, the Koszul complex in Section 3.1 is not always acyclic. Hence, the symmetry of the number may break down in the non-CohenMacaulay case. So we have the problem as to what structure the local cohomology (resp. the solution sheaf) of $\mathcal{F} M_{A}(\boldsymbol{\beta})$ has in the non-Cohen-Macaulay case. This remains unsettled.

\section{REFERENCES}

[1] A. Adolphson, Hypergeometric functions and rings generated by monomials, Duke Math. J. 73 (1994), 269-290.

[ 2 ] A. Adolphson, Higher solutions of hypergeometric systems and Dwork cohomology, Rend. Sem. Mat. Univ. Padova 101 (1999), 179-190.

[ 3 ] A. Borel, et al., Algebraic $D$-modules, Perspect. Math. 2, Academic Press, Boston, Mass., 1987.

[ 4 ] W. Bruns And J. Herzog, Cohen-Macaulay Rings, Cambridge Stud. Adv. Math. 39, Cambridge University Press, Cambridge, 1993

[ 5 ] W. Fulton, Introduction to toric varieties, Ann. of Math. Stud. 131, Princeton University Press, Princeton, N.J., 1993.

[6] I. M. Gel'Fand, M. M. Kapranov and A. V. Zelevinski, Generalized Euler integrals and Ahypergeometric functions, Adv. Math. 84 (1990), 255-271.

[ 7 ] I. M. Gel'fand, A. V. Zelevinski AND M. M. KapRanov, Hypergeometric functions and toric varieties, Funct. Anal. Appl. 23 (1989), 94-106.

[ 8 ] R. HоTTA, Equivariant $D$-modules, arXiv. math.RT/9805021 (1998).

[ 9 ] R. Hotta And M. Kashiwara, The invariant holonomic system on a semisimple Lie algebra, Invent. Math. 75 (1984), 327-358.

[10] R. Hotta And T. TAnis aKi, D-modules and Algebraic Groups, Springer, Tokyo, 1995 (in Japanese).

[11] J. McCleary, User's guide to spectral sequences, Math. Lecture Ser. 12, Publish or Perish, Wilmington, Del., 1985.

[12] M. SAITo, Isomorphism classes of A-hypergeometric systems, Composito Math. 128 (2001), 323-338.

[13] M. Saito And W. N. Traves, Differential algebras on semigroup algebras, Symbolic Computation: solving equations in algebra, geometry, and engineering (South Hadley, MA, 2000), 207-226, Comtemp. Math. 286, Amer, Math. Soc., Providence, R.I., 2001.

[14] M. Saito, B. Sturmfels and N. TAKayama, Gröbner deformations of hypergeometric differential equations, Algorithms Comput. Math. 6, Springer, Berlin, 2000.

HOKKAIDO INSTITUTE OF TECHNOLOGY

SAPPORO 006-8585

JAPAN

E-mail address: gokuyama@hit.ac.jp 